\title{
The Impact of the Introduction of Total Mesorectal Excision on Local Recurrence Rate and Survival in Rectal Cancer: Long-Term Results
}

\author{
C. A. Maurer, MD, FRCS, FACS ${ }^{1,2}$, P. Renzulli, MD ${ }^{1}$, C. Kull, MD $^{2}$, S. A. Käser, MD ${ }^{2}$, L. Mazzucchelli, MD ${ }^{3}$, \\ A. Ulrich, $\mathrm{MD}^{4}$, and M. W. Büchler, MD, $\mathrm{FRCS}^{1,4}$ \\ ${ }^{1}$ Department of Visceral and Transplantation Surgery, University of Berne, Berne, Switzerland; ${ }^{2}$ Department of General, \\ Visceral, Vascular and Thoracic Surgery, Hospital of Liestal, Liestal, Switzerland; ${ }^{3}$ Institute of Pathology, University of \\ Berne, Berne, Switzerland; ${ }^{4}$ Department of General Visceral and Transplantation Surgery, University of Heidelberg, \\ Heidelberg, Germany
}

\begin{abstract}
Purpose. To investigate the influence of the introduction of total mesorectal excision (TME) on local recurrence rate and survival in patients with rectal cancer.

Methods. A total of 171 consecutive patients underwent anterior or abdominoperineal resection for primary rectal cancer. When the TME technique was introduced, the clinical setting, including the surgeons, remained the same. Group 1 (1993-95, $n=53)$ underwent conventional surgery and group $2(1995-2001, n=118)$ underwent TME. All patients were followed for 7 years or until death.

Results. Between the two groups, no statistically significant differences were present with regards to patient-, treatment-, or tumor-related characteristics apart from the time point of radiotherapy. The total local recurrence rates were 11 of $53(20.8 \%)$ in group 1 and 7 of 118 (5.9\%) in group 2, and the rates of isolated local recurrences were 6 of $53(11.3 \%)$ in group 1 and 2 of $118(1.7 \%)$ in group 2. Both differences were highly statistically significant. The disease-free survival in groups 1 and 2 was 60.4 and $65.3 \%$ at 5 years, and 58.5 and $65.3 \%$ at 7 years, respectively. Excluding patients with synchronous or metachronous distant metastasis from the analysis, both the disease-free survival and the cancer-specific survival were statistically
\end{abstract}

C. A. Maurer and P. Renzulli contributed equally to this work.

(C) Society of Surgical Oncology 2011

First Received: 2 May 2010;

Published Online: 5 February 2011

C. A. Maurer, MD, FRCS, FACS

e-mail: christoph.maurer@ksli.ch significantly better in group 2 than in group 1. No statistically significant difference between the two groups was detected regarding the overall survival.

Conclusions. The introduction of TME led to an impressive reduction of the local recurrence rate. Survival is mainly determined by the occurrence of distant metastasis, but TME seems to improve survival in patients without systemic disease.

Total mesorectal excision (TME) has become a widely accepted modality for major resection of rectal cancer. ${ }^{1,2}$ The technique of TME has been described in detail previously, and its principles should be familiar to every surgeon currently performing rectal cancer surgery. ${ }^{3,4}$ No randomized studies are available that compare conventional rectal cancer surgery with TME, most probably as a result of the obvious advantages of TME. Several specialized centers reported in their TME series a local recurrence rate of below $10 \%$, whereas conventional rectal cancer surgery has local recurrence rates of $20-30 \% .^{5-9}$ However, little is known about the impact of introduction of TME in an established surgical team that performed conventional rectal cancer surgery and then switched to TME. ${ }^{10,11}$

The influence of TME on the oncological long-term outcome has, so far, been poorly investigated. Most of the TME studies report a median follow-up of 5 years or less. ${ }^{711-15}$ However, an analysis of 169,658 French patients with colorectal cancer who were initially treated with curative intent estimated that the time to cure is 9.3 years. ${ }^{16}$ Similarly, a Japanese group found cumulative recurrence rates in rectal cancer of $89 \%$ at 5 years, $98 \%$ at 
7 years, and $100 \%$ at 10 years. ${ }^{17}$ Therefore, any reliable statement with regard to recurrence and survival in rectal cancer needs a minimum follow-up of 7 years for every patient operated with curative intent. This might be especially true for TME patients because the potentially residual local tumor burden is estimated to be small and therefore might need time to manifest as local recurrence.

There are a number of further issues in the current literature on TME. For example, studies on TME written in Chinese are difficult to read by nonnative speakers. ${ }^{18}$ Some studies were confounded by irradiation. ${ }^{19,20}$ In some, the analyzed patients originated from randomized trials with other study aims or from highly specialized institutes, which might be biased. ${ }^{5,6,21,22}$ There are only a few population- and surgeon-based reports on the influence of introduction of TME, although they are probably the best reflection of daily practice. ${ }^{15,23}$ One of them, by performing a subgroup analysis, showed a survival advantage after introduction of TME, but no data on local recurrence rate were available. ${ }^{23}$ The limit of another study was that the follow-up ended after 5 years. ${ }^{15}$

The present study aimed to investigate the impact of the introduction of TME on the reduction of the local recurrence rate in a stable clinical setting, meaning the same surgical staff, medical institution, and population. In addition, because the follow-up for every one of our patients with resected rectal cancer was at least 7 years, this study is a reliable statement of the impact of TME on long-term outcome.

\section{PATIENTS AND METHODS}

Within the period of the last author's chairmanship (M.W.B.) at the University Hospital of Berne, from November 1993 until October 2001, a total of 194 patients with rectal cancer underwent surgery in his department. Rectal cancer was defined as histologically proven adenocarcinoma at or below $15 \mathrm{~cm}$ from the anal verge, measured with a rigid rectoscope. The data from all patients were consecutively and prospectively recorded in a computerized database.

Thirteen of 194 patients had no resection of the primary due to locally or systemically far advanced disease (nine patients with International Union Against Cancer [UICC] stage IV disease and four patients with stage III disease and/or high operative risk), giving a resectability rate of 93.3\%. Seven patients refused regular follow-up or were lost. Three patients had local excision of an early cancer via a transanal or a posterior approach. The patients of these 3 subgroups were excluded from further analysis, leaving a study population of 171 patients with low anterior resection or abdominoperineal resection and complete follow-up. These 171 patients were further divided into two groups, one before the introduction of the TME technique (group 1, from November 1993 until July 1995) and the other after (group 2, from July 1995 until October 2001).

Patient-, treatment-, and tumor-related characteristics of the total study collective as well as of groups 1 and 2 separately are listed in detail in Table 1. Patients with rectal cancer of stage II or III according to UICC criteria were recommended to undergo (neo)adjuvant radiochemotherapy. Exclusions for adjuvant treatment were protracted infectious complications of the tumor or of the surgery, and usually the tumor site within the upper third of rectum. The neoadjuvant treatment consisted of shortcourse radiotherapy $(5 \times 5$ Gy per week) followed by immediate surgery if the aim of radiotherapy was sterilization of intrapelvic micrometastasis, or radiotherapy 45-50.4 Gy per 5-6 weeks combined with 5-fluorouracil infusion followed by surgery with a delay of 4-6 weeks if the primary aim of radiotherapy was to downsize the intrapelvic tumor. The schedule of the postoperative radiotherapy was $45-50.4$ Gy over 5-6 weeks, applied in single doses of $1.8 \mathrm{~Gy}$, and combined with 5-fluorouracil infusion.

The conventional rectal excision (group 1) consisted of a dissection of the superior rectal artery or the inferior mesenteric artery and further preparation toward the pelvis directly on the aortic bifurcation and the common iliac arteries. Mobilization of the rectum at the dorsal aspect was done bluntly by the surgeon's hand or fingers, whereas the mesorectum laterally on both sides was sharply dissected between clamps and suture stitches. Anteriorly, either sharp or blunt dissection was used. No attention was paid to preserve the autonomic nerve structures.

The TME technique as described by Heald et al. was introduced in our institution in July $1995 .{ }^{3}$ For this, the staff surgeons from our department who usually performed rectal resections visited Bill Heald in Basingstoke to watch him perform TME. They learned the TME technique from his videos and from several interactive workshops with him.

Briefly, complete removal of the mesorectum and the mesentery containing the inferior mesenteric artery and vein and the main locoregional lymphatic system of the rectum could be achieved by meticulous sharp dissection of the avascular plane, the so-called holy plane, between parietal and visceral pelvic fascia. ${ }^{24}$ Anteriorly, the specimen included the intact Denonvilliers fascia and the peritoneal reflexion. Autonomic nerve structures were preserved. Little autonomic nerves branching off the inferior hypogastric plexus directly into the rectum as well as the middle rectal artery, if present, needed to be cut. No clamps were used to control blood vessels. The resection plane was extended laterally in locally advanced rectal tumors only if tumorous adherence or infiltration of the 
TABLE 1 Patient-, treatment-, and tumor-related characteristics

\begin{tabular}{|c|c|c|c|c|}
\hline Characteristic & $\begin{array}{l}\text { Total } \\
(n=171)\end{array}$ & $\begin{array}{l}\text { Group } 1 \text { (conventional) } \\
(n=53)\end{array}$ & $\begin{array}{l}\text { Group } 2(\mathrm{TME}) \\
(n=118)\end{array}$ & $\begin{array}{l}P \text { (group } 1 \text { vs. } \\
\text { group 2) }\end{array}$ \\
\hline Sex & & & & 0.35 \\
\hline Female & 59 & 21 & 38 & \\
\hline Male & 112 & 32 & 80 & \\
\hline Median (range) age at operation (year) & $65.6(33.4-89.6)$ & $64.5(33.4-85.4)$ & $66.8(34.4-89.6)$ & 0.29 \\
\hline $\begin{array}{l}\text { Median (range) tumor diameter }(\mathrm{cm}) \text {, measured } \\
\text { after fixation in formalin }\end{array}$ & $4.0(0.5-9.5)$ & $4.5(0.5-9.5)$ & $4.0(0.5-9.0)$ & 0.24 \\
\hline Median (range) distance of tumors from anal verge $(\mathrm{cm})$ & $8.0(1.0-15.0)$ & $8.0(2.0-15.0)$ & $8.0(1.0-15.0)$ & 0.62 \\
\hline Type of operation & & & & 0.38 \\
\hline (Low) anterior resection, $n(\%)$ & $142(83.0)$ & $42(79.3)$ & $100(84.7)$ & \\
\hline Abdominoperineal resection, $n(\%)$ & $29(17.0)$ & $11(21.7)$ & $18(15.3)$ & \\
\hline UICC tumor stage & & & & 0.33 \\
\hline $\mathrm{I}$ & $53(31.0)$ & $18(34.0)$ & $35(29.7)$ & \\
\hline II & $31(18.1)$ & $13(24.5)$ & $18(15.3)$ & \\
\hline III & $51(29.8)$ & $12(22.6)$ & $39(33.1)$ & \\
\hline IV & $36(21.1)$ & $10(18.9)$ & $26(22.0)$ & \\
\hline Preoperative radiotherapy, $n(\%)$ & $19(11.1)$ & $1(1.8)$ & $18(15.3)$ & 0.01 \\
\hline Postoperative radiotherapy, $n(\%)$ & $16(9.4)$ & $9(17.0)$ & $7(5.9)$ & 0.02 \\
\hline Total irradiated patients, $n(\%)$ & $35(20.5)$ & $10(18.9)$ & $25(21.2)$ & 0.73 \\
\hline Postoperative chemotherapy, $n(\%)$ & $66(38.6)$ & $19(35.8)$ & $47(39.8)$ & 0.62 \\
\hline
\end{tabular}

UICC International Union Against Cancer

visceral pelvic fascia occurred, always with the goal of obtaining a clear lateral resection margin. Before dissection of the rectum distally, a rectangle clamp was placed on the mobilized rectum distally to the tumor, and a rectal stump washout was performed. ${ }^{25}$ Both manoeuvres should help to avoid tumor cell contamination and consecutive tumor seeding. Within group 2, all tumors of the middle or lower third of the rectum were treated with a TME; tumors of the upper third were removed by a (longitudinally) partial mesorectal excision. Care was taken to preserve the pelvic autonomic nerve structures. ${ }^{26}$

Colorectal or coloanal reconstruction was performed by using the descending or transverse colon as a straight colonic section, as a colon $\mathrm{J}$ pouch, or as a transverse coloplasty pouch. The transverse coloplasty pouch was developed in an animal model by our group and was later adopted in humans. ${ }^{27,28}$ All anastomoses at or below $6 \mathrm{~cm}$ from the anal verge were protected by a temporary loop ileostomy. All patients received a preoperative orthograde bowel preparation. An omental patch was placed into the sacral cavity to reduce the risk of pelvic abscesses. In group 2, the TME technique was also used for patients needing an abdominoperineal resection. In both groups, abdominoperineal resection was performed without wide excision of the anal sphincter muscle and the pelvic floor. Indications for abdominoperineal resection were tumorous infiltration of the external anal sphincter muscle and/or the puborectal sling, if known from pretreatment investigations or detected intraoperatively.

Early in 1997, our pathologists started to investigate the circumferential resection margin according the method published by Quirke et al. ${ }^{29,30}$ In brief, the rectal specimen including the mesorectum were sent unopened to pathology. The circumferential resection margin of the mesorectum were marked with ink. After fixation in formalin, the specimen was then cut in 5-mm slices and documented by photographs. From sites where the tumor grossly reached near the circumferential resection margin or where the mesorectal fascia was damaged, a meticulous microscopic workup was performed. An R1 resection was defined as the distance between the cancer and the circumferential or distal resection margin of less than $1 \mathrm{~mm} .{ }^{30}$

Patients were in a regular surveillance program according to the guidelines of the Swiss Society of Gastroenterology established in 1996 and slightly modified in 2001, 2004, and 2007. ${ }^{31}$ Before 1996, our own and even more intensive follow-up schedule was used. All versions recommended at least the following examinations: clinical examination and serum carcinoembryonic antigen measurement every 6 months during the first 2 years and yearly thereafter; rectosigmoidoscopy and rectal endosonography (if available) every 6 months during the first 2 years; complete colonoscopy after 3 or 4 years, then every 5 years; and liver ultrasound or thoracoabdominal 
computed tomographic scan annually for 3 years. Examinations were intensified if recurrence was suspected. In addition, the follow-up status of the patients regarding recurrence, sites of recurrence, time to recurrence, survival, and time of death were assessed by standardized reimbursed questionnaires sent to the treating physician or oncologist, or by telephone conversations with these colleagues. All patients were followed for at least 7 years after resection of their rectal cancer or until death.

The trial was approved by the local ethical committee (KEK No. 08-05-09). It was registered at ClinicalTrials.gov, identification no. NCT00910143.

Results are expressed as means including standard errors of mean or medians including the range. Differences between the two groups (conventional surgery vs. TME) regarding age, tumor diameter, distance of tumor from the anal verge, distal safety margin, number of investigated lymph nodes, and number of positive lymph nodes (quantitative data) were analyzed with a Mann-Whitney $U$-test. Differences in the remaining patient, treatment, and tumor characteristics (category data) in both groups were calculated by the $\chi^{2}$ test. The importance of the factors influencing local recurrence was compared by logistic regression analysis. Survival analyses were performed by the Kaplan-Meier method, and the importance of the factors influencing disease-free and overall survival was compared by multivariate regression analysis. The statistical significance was attributed at the 5\% level. WinStat version 5.3 and STATA 10.0 served as the computerized statistical analysis systems.

\section{RESULTS}

The percentage of sphincter-saving procedures in the whole series was $83.0 \%$. One postoperative death occurred at day 9 from cardiac arrest in an 82-year-old patient in group 2.

The specific results related to surgical quality and oncosurgical radicality of the total study collective as well as of groups 1 and 2 are summarized in Table 2. According to the method of Kaplan--Meier, the long-term outcomes of both groups are depicted as disease-free survival (Fig. 1), as overall survival (Fig. 2), as cancer-specific survival (Fig. 3), and as cancer-specific survival excluding patients with synchronous or metachronous distant metastases (Fig. 4).

The disease-free survival excluding synchronous and metachronous distant metastases was significantly better in the TME group $(P=0.02)$.

Logistic regression analysis showed that the use of TME $(P=0.001), \mathrm{R} 0$ resection $(P=0.003$,$) and negative nodal$ stage $(P=0.028)$ significantly reduced local recurrence rate. Local tumor stage of $<\mathrm{T} 3(P=0.084)$, age $(P=$ $0.931)$, male sex $(P=0.370)$, tumor diameter $(P=$ $0.954)$, security distance $(P=0.296)$, distance to the anal verge $(P=0.966)$, neoadjuvant therapy $(P=0.120)$, adjuvant chemotherapy $(P=0.198)$, and adjuvant radiotherapy $(P=0.134)$ did not have a significant impact on the local recurrence rate.

Multivariate regression analysis showed that age $(P=0.000)$ and UICC tumor stage IV $(P=0.000)$

TABLE 2 Results for surgical quality, oncosurgical radicality, and long-term outcome

\begin{tabular}{|c|c|c|c|c|}
\hline Characteristic & $\begin{array}{l}\text { Total } \\
(n=171)\end{array}$ & $\begin{array}{l}\text { Group } 1 \text { (conventional) } \\
(n=53)\end{array}$ & $\begin{array}{l}\text { Group } 2(\mathrm{TME}) \\
(n=118)\end{array}$ & $\begin{array}{l}P \text { (group } 1 \text { vs. } \\
\text { group 2) }\end{array}$ \\
\hline Septic complications within the pelvis, $n(\%)$ & $9(5.7)$ & $3(5.7)$ & $6(5.1)$ & 0.88 \\
\hline $\begin{array}{l}\text { Median (range) distal safety margin }(\mathrm{cm}) \text {, measured } \\
\text { after fixation in formalin }\end{array}$ & $3(0.1-10.0)$ & $3(0.5-8.0)$ & $3(0.1-10.0)$ & 0.24 \\
\hline \multicolumn{5}{|l|}{ Residual tumor (locoregional) } \\
\hline $\mathrm{R} 1$ resection, $n(\%)$ & $9(5.7)$ & $4(7.5)$ & $5(4.2)$ & 0.37 \\
\hline Local R2 resection, $n(\%)$ & $2(1.2)$ & 0 & $2(1.7)$ & 0.34 \\
\hline Median (range) no. of investigated nodes & $12(1-69)$ & $9(1-22)$ & $13(1-69)$ & $<0.001$ \\
\hline $\begin{array}{l}\text { Median (range) no. of positive nodes in node } \\
\text { positive patients }\end{array}$ & $3.0(1-20)(n=78)$ & $2.5(1-10)(n=18)$ & $3.0(1-20)(n=60)$ & 0.42 \\
\hline \multicolumn{5}{|l|}{ Recurrence } \\
\hline Local recurrence, total $n(\%)$ & $18(10.5)$ & $11(20.8)$ & $7(5.9)$ & 0.003 \\
\hline Local recurrence, only $n(\%)$ & $8(4.7)$ & $6(11.3)$ & $2(1.7)$ & 0.006 \\
\hline Distant metastasis, total, $n(\%)$ & $59(34.5)$ & $17(32.1)$ & $42(35.6)$ & 0.65 \\
\hline Distant metastasis, only, $n(\%)$ & 49 (28.7) & $12(22.6)$ & $37(31.4)$ & 0.24 \\
\hline Disease-free survival after 5 years & $63.7 \%$ & $60.4 \%$ & $65.3 \%$ & 0.60 \\
\hline Disease-free survival after 7 years & $63.2 \%$ & $58.5 \%$ & $65.3 \%$ & 0.45 \\
\hline
\end{tabular}

UICC International Union Against Cancer, TME total mesorectal excision, R1 microscopic residual tumor (at resection margin), R2 gross (macroscopic) residual tumor 




FIG. 1 Disease-free survival

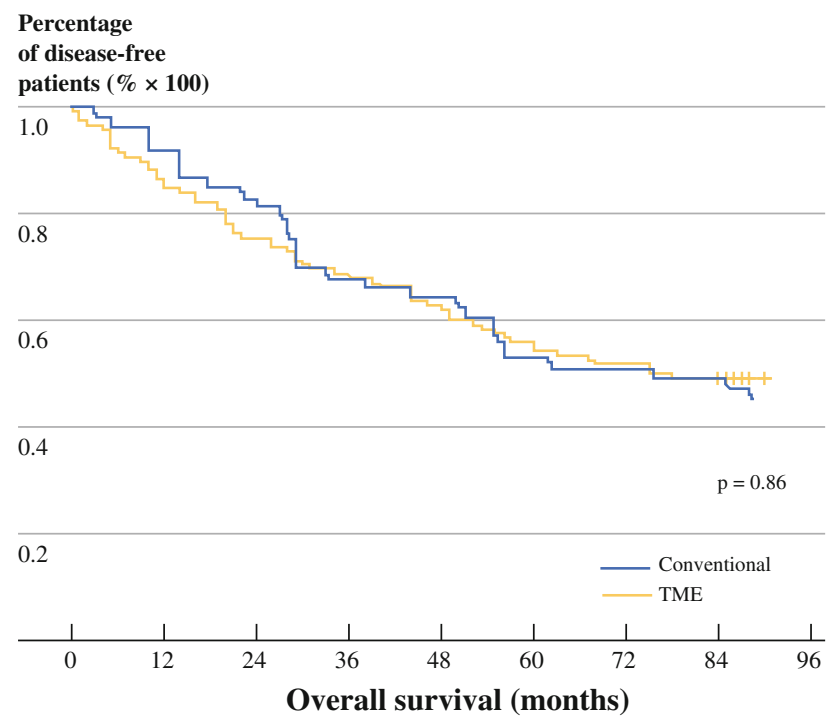

FIG. 2 Overall survival

significantly reduced overall survival, whereas the following factors had no significant impact on overall survival: UICC tumor stage III $(P=0.070)$, UICC tumor stage II $(P=0.220)$, UICC tumor stage I $(P=0.224)$, distance to the anal verge $(P=0.811)$, tumor diameter $(P=0.957)$, the use of low anterior rectum resection versus abdominoperineal amputation $(P=0.490)$, the use of TME $(P=0.483)$, R0 resection $(P=0.659)$, distant margin $(P=0.127)$, perforation during operation $(P=0.325)$, pelvic abscess $(P=0.965)$, neoadjuvant therapy $(P=0.373)$, adjuvant chemotherapy $(P=0.675)$, adjuvant radiotherapy $(P=0.345)$, male sex $(P=0.121)$, and duration of hospitalization $(P=0.941)$.

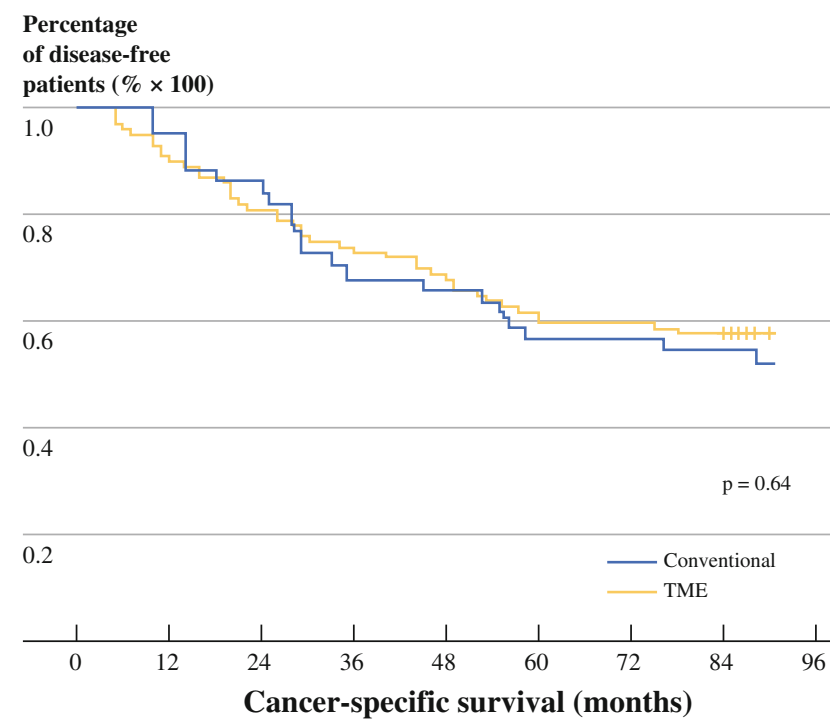

FIG. 3 Cancer-specific survival

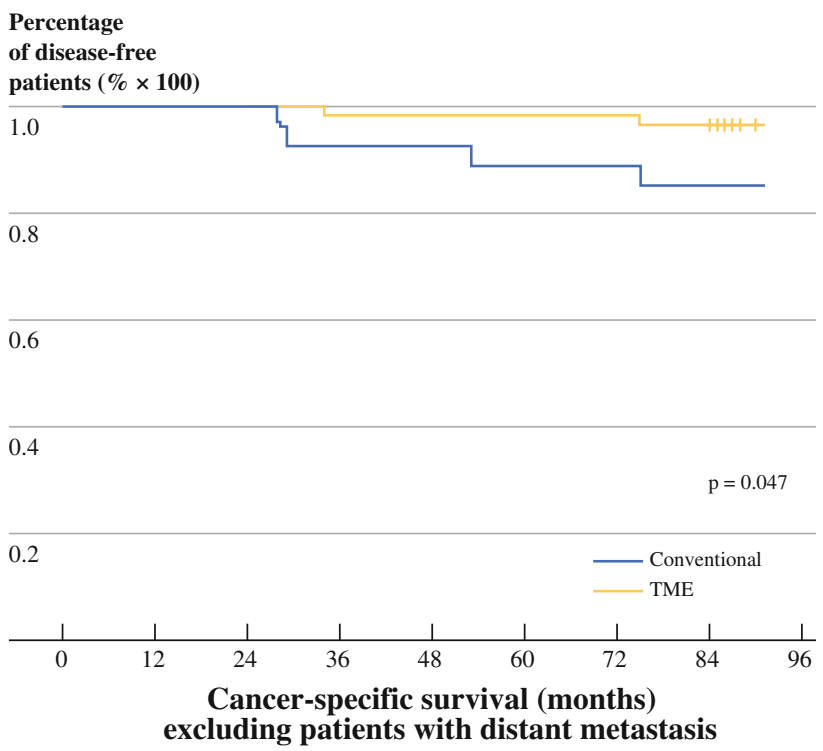

FIG. 4 Cancer-specific survival excluding patients with distant metastasis

Regarding disease-free survival, multivariate regression analysis showed that age $(P=0.003)$, UICC tumor stage III $(P=0.018)$, and UICC tumor stage IV $(P=0.000)$ significantly reduced disease-free survival, whereas all the other factors had no significant impact on disease-free survival: UICC tumor stage II $(P=0.177)$ and UICC tumor stage I $(P=0.250)$, distance to the anal verge $(P=0.335)$, tumor diameter $(P=0.635)$, the use of low anterior rectum resection versus abdominoperineal amputation $(P=0.979)$, the use of TME $(P=0.213)$, R0 resection $(P=0.533)$, distant margin $(P=0.051)$, perforation during operation $(P=0.420)$, pelvic abscess $(P=0.866)$, neoadjuvant therapy $(P=0.676)$, adjuvant 
chemotherapy $(P=0.602)$, adjuvant radiotherapy $(P=$ $0.0621)$, male sex $(P=0.056)$, and duration of hospitalization $(P=0.853)$.

When we analyzed the potential impact of (neo)adjuvant radiotherapy plus chemotherapy for patients with tumor stages II and III, we found that the local recurrence rates did not seem to improve by means of such a standard treatment: in the conventional group, 4 out of 17 (23.5\%) patients not treated according to a standard protocol had local recurrence, versus 3 of $8(37.5 \%)$ patients treated, and 3 of $48(6.3 \%)$ not treated versus 1 of $9(11.1 \%)$ treated in the TME group, respectively. When we stopped the survival analysis in both groups at 7 years or earlier death, the mean disease-free survival in the conventional group was 49 months without standard therapy and 53 months with standard therapy, and in the TME group 58 months without standard therapy and 55 months with standard therapy.

In the conventional group, two of six patients with isolated local recurrence could successfully undergo repeat operation without developing a repeat recurrence, whereas in the TME group, both patients with isolated local recurrence died from it.

\section{DISCUSSION}

In a consecutive series of patients with rectal cancer, the impact of the introduction of TME on pelvic recurrence and survival was investigated. Both groups of patients, the conventionally operated group and the TME group originated from the same population and were treated by the same staff of surgeons within the same institution. In the present series, the introduction of TME led to a highly statistically significant decrease of the local recurrence rate, from $20.8 \%$ in the conventional surgery group to $5.9 \%$ in the TME group. Thus TME reduced the number of local recurrences to below one third. Given the long follow-up period of at least 7 years or until death for all patients, these figures are reliable because more than $85 \%$ of all local recurrences become detectable within 3 years after primary surgery, and a recurrence-free period of 7 years is regarded as a definite cure in more than $98 \%$ of colorectal carcinomas. $^{11,16,17}$

The analysis of patients with local recurrence only, as sole site of recurrence, revealed local recurrence rates of $11.3 \%$ in the conventional group and $1.7 \%$ in the TME group. Again, this surgical progress is highly statistically significant and of the utmost importance because surgical salvage procedures-if feasible at all—may be demanding, mutilating, expensive, and ultimately not curative. ${ }^{32}$ Many surgeons no doubt still have patients in mind who slowly deteriorated over the course of years with painfully invading local recurrence of rectal cancer.
Both groups showed similar patient and tumor characteristics, with the exception that radiotherapy was performed more often postoperatively in the conventional group and more often preoperatively in the TME group. This is a limitation of the study because preoperative radiotherapy might have induced size reduction of tumors and facilitated the dissection. One might argue that this shift from mainly postoperative to preoperative radiotherapy might have substantially contributed to the drop in local recurrence rate in the TME group. Indeed, two randomized trials have demonstrated an advantage for the preoperative irradiation modus. ${ }^{33,34}$ By application of irradiation to every patient in these studies, a reduction in local recurrence rates in the preoperatively irradiated patients of 9 and $7 \%$, respectively, was observed. ${ }^{33,34} \mathrm{In}$ the present study, however, only $20 \%$ of all patients received any radiotherapy at all. Therefore, the optimized time point of irradiation in the TME group is estimated to count for less than $2 \%$ reduction of the local recurrence rate and may not explain the reduction of $15 \%$ found in our study. Furthermore, the potential confounding factor of different time points of radiotherapy was weakened by logistic regression analysis, which showed TME to be the most important factor in preventing local recurrence.

Despite the impressive reduction of the local recurrence rate in the TME group, surprisingly, no statistically significant difference in disease-free or overall survival could be detected between the two groups. Several circumstances specific to the present study may be responsible for this fact. First, the initially advanced UICC tumor stages III and IV were more frequent in the TME group than in the conventional group. Second, in the TME group, the total proportion of patients with synchronous or metachronous distant metastasis exceeded the corresponding proportion in the conventional group by $3.5 \%$. Finally, patients in the TME group were a median of 2.3 years older than in the conventional group, leading to a possible negative impact on overall survival. Indeed, $24.3 \%$ of the recurrence-free patients died from another cause in the TME group during the 7-year follow-up period, but only $20.0 \%$ did so in the conventional group. These factors, taken together, might have negatively influenced survival in the TME group compared with the conventional group. However, in the multivariate analysis, TME did not turn out to be a statistically significant predictive factor of disease-free or overall survival. Nevertheless, the TME group showed a $5 \%$ benefit for 5 -year disease-free survival and a $7 \%$ benefit for 7-year disease-free survival (Table 2, Fig. 1). These differences might have become significant in a study with a larger sample size, but the study had to be stopped at an underpowered state of accrual because the first and last authors departed the surgical department, which was equivalent to a change in the study setting. ${ }^{23}$ Further, 
omission of (neo)adjuvant therapy in most patients with stage II or III disease did not seem to be responsible for the lack of improvement in survival of the TME group.

However, even these rather small disease-free and cancer-specific survival benefits in the TME group, when compared to the conventional group, should support the TME technique further because the beneficial effect of TME in our study is almost as high as that of adjuvant systemic chemotherapies. Furthermore, excluding patients with synchronous or metachronous distant metastasis from the analysis, both the disease-free survival and the cancerspecific survival were statistically significantly better in the TME group than in the conventional group.

Although not directly comparable, the Dutch TME trial and other studies also failed to show an overall survival benefit, despite an improved rate of local recurrence. ${ }^{15,21,34}$ Survival seems to be mainly determined by the occurrence of distant disease. Laurent et al. found that patients with postoperative pelvic sepsis had a 5-year survival rate of $39 \%$ compared with $65 \%$ without pelvic sepsis. ${ }^{7}$ They hypothesized that the expected survival benefit by TME is neutralized by an increased pelvic sepsis rate that is associated with increased risk of distant recurrence and decreased long-term survival. In the present study, however, a similarly low pelvic sepsis rate was encountered in both groups, probably facilitated by the consequent use of a pelvic omental patch and a defunctioning loop ileostomy for all anastomoses below $7 \mathrm{~cm}$ from the anal verge.

In conclusion, within a stable study setting, the introduction of TME led to a clear reduction of the local recurrence rate in rectal cancer. After TME, local recurrences occurred exceptionally as the sole site of recurrence but were almost always accompanied by distant metastasis. The long-term follow-up of 7 or more years for all patients revealed only a tendential survival benefit from TME, probably as a result of a higher number of adverse factors in group 2 than in group 1. Future therapeutic efforts should be directed to control recurrences from distant metastasis.

ACKNOWLEDGMENT The data management (in part) and a specialist English-language editing service were funded by the fund for surgical education and research of our department, accepted by the chief executive officer of our hospital. We thank the data manager, Miriam Kull, from Liestal, for her help in assessing some of the follow-up data.

\section{REFERENCES}

1. Heald RJ. Total mesorectal excision is optimal surgery for rectal cancer: a Scandinavian consensus. Br J Surg. 1995;82:1297-9.

2. Mella J, Biffin A, Radcliffe AG, Stamatakis JD, Steele RJ. Population-based audit of colorectal cancer management in two UK health regions. Colorectal Cancer Working Group, Royal
College of Surgeons of England Clinical Epidemiology and Audit Unit. Br J Surg. 1997;84:1731-6.

3. Heald RJ, Husband EM, Ryall RD. The mesorectum in rectal cancer surgery-the clue to pelvic recurrence? Br J Surg. 1982;69:613-6.

4. Maurer CA, Renzulli P, Meyer JD, Büchler MW. Rectal carcinoma. Optimizing therapy by partial or total mesorectum removal. Zentralbl Chir. 1999;124:428-35.

5. Heald RJ, Moran BJ, Ryall RD, Sexton R, MacFarlane JK. Rectal cancer: the Basingstoke experience of total mesorectal excision, 1978-1997. Arch Surg. 1998;133:894-9.

6. Enker WE, Merchant N, Cohen AM, et al. Safety and efficacy of low anterior resection for rectal cancer: 681 consecutive cases from a specialty service. Ann Surg. 1999;230:544-52.

7. Laurent C, Nobili S, Rullier A, Vendrely V, Saric J, Rullier E. Efforts to improve local control in rectal cancer compromise survival by the potential morbidity of optimal mesorectal excision. J Am Coll Surg. 2006;203:684-91.

8. Gunderson LL, Sargent DJ, Tepper JE, et al. Impact of T and N stage and treatment on survival and relapse in adjuvant rectal cancer: a pooled analysis. J Clin Oncol. 2004;22:1785-96.

9. Hermanek P, Wiebelt H, Staimmer D, Riedl S. Prognostic factors of rectum carcinoma-experience of the German Multicentre Study SGCRC. German Study Group colo-rectal carcinoma. Tumori. 1995;81:60-4.

10. Kapiteijn E, Putter H, van de Velde CJ; Cooperative Investigators of the Dutch ColoRectal Cancer Group. Impact of the introduction and training of total mesorectal excision on recurrence and survival in rectal cancer in The Netherlands. Br J Surg. 2002; 89:1142-9.

11. Bernardshaw SV, Øvrebø K, Eide GE, Skarstein A, Røkke O. Treatment of rectal cancer: reduction of local recurrence after the introduction of TME- experience from one university hospital. Dig Surg. 2006;23:51-9.

12. Tilney HS, Tekkis PP. Extending the horizons of restorative rectal surgery: intersphincteric resection for low rectal cancer. Colorectal Dis. 2008;10:3-15.

13. Wu AW, Gu J, Wang J, et al. Results after change of treatment policy for rectal cancer-report from a single hospital in China. Eur J Surg Oncol. 2007;33:718-23.

14. Frileux P, Burdy G, Aegerter P, et al. Surgical treatment of rectal cancer: results of a strategy for selective preoperative radiotherapy. Gastroenterol Clin Biol. 2007;31:934-40.

15. Visser O, Bakx R, Zoetmulder FA, et al. The influence of total mesorectal excision on local recurrence and survival in rectal cancer patients: a population-based study in Greater Amsterdam. J Surg Oncol. 2007;95:447-54.

16. Chauvenet M, Lepage C, Jooste V, Cottet V, Faivre J, Bouvier AM. Prevalence of patients with colorectal cancer requiring follow-up or active treatment. Eur J Cancer. 2009;45:1460-5.

17. Sadahiro S, Suzuki T, Ishikawa K, et al. Recurrence patterns after curative resection of colorectal cancer in patients followed for a minimum of ten years. Hepatogastroenterology. 2003;50:1362-6.

18. Liang YC, Qing SH, Ding WX, Chen PY, Huang YB, Yu HT. Total mesorectal excision versus conventional radical surgery for rectal cancer: a meta analysis (in Chinese). Zhonghua Wei Chang Wai Ke Za Zhi. 2007;10:43-8.

19. den Dulk M, Krijnen P, Marijnen CA, et al. Improved overall survival for patients with rectal cancer since. 1990: The effects of TME surgery and pre-operative radiotherapy. Eur J Cancer. 2008;44:1710-6.

20. Hansen MH, Kjaeve J, Revhaug A, Eriksen MT, Wibe A, Vonen B; Norwegian Rectal Cancer Group. Impact of radiotherapy on local recurrence of rectal cancer in Norway. Br J Surg. 2007; 94:113-8. 
21. Peeters KC, Marijnen CA, Nagtegaal ID; Dutch Colorectal Cancer Group, et al. The TME trial after a median follow-up of 6 years: increased local control but no survival benefit in irradiated patients with resectable rectal carcinoma. Ann Surg. 2007;246:693-701.

22. Påhlman L. Population based study of factors influencing occurrence and prognosis of local recurrence after surgery for rectal cancer. Tech Coloproctol. 2003;7:120-1.

23. Dahlberg M, Påhlman L, Bergström R, Glimelius B. Improved survival in patients with rectal cancer: a population-based register study. Br J Surg. 1998;85:515-20.

24. Heald RJ. The "holy plane" of rectal surgery. J $R$ Soc Med. 1988;81:503-8.

25. Constantinides VA, Cheetham D, Nicholls RJ, Tekkis PP. Is rectal washout effective for preventing localized recurrence after anterior resection for rectal cancer? Dis Colon Rectum. 2008;51:1339-44.

26. Maurer CA, Z'Graggen K, Renzulli P, Schilling MK, Netzer P, Büchler MW. Total mesorectal excision preserves male genital function compared with conventional rectal cancer surgery. $\mathrm{Br} \mathrm{J}$ Surg. 2001;88:1501-5.

27. Maurer CA, Z'graggen K, Zimmermann W, Häni HJ, Mettler D, Büchler MW. Experimental study of neorectal physiology after formation of a transverse coloplasty pouch. $\mathrm{Br} J$ Surg. 1999;86:1451-8.

28. Z'graggen K, Maurer CA, Birrer S, Giachino D, Kern B, Büchler MW. A new surgical concept for rectal replacement after low anterior resection: the transverse coloplasty pouch. Ann Surg. 2001;234:780-5.

29. Quirke P, Durdey P, Dixon MF, Williams NS. Local recurrence of rectal adenocarcinoma due to inadequate surgical resection. Histopathological study of lateral tumour spread and surgical excision. Lancet. 1986;2:996-9.

30. Nagtegaal ID, Quirke P. What is the role for the circumferential margin in the modern treatment of rectal cancer? J Clin Oncol. 2008;26:303-12.

31. Swiss Society of Gastroenterology. Revised consensus recommendations for surveillance of colorectal cancer surgically treated in curative intent. http://www.sggssg.ch/Dokumente/ Merkblaetter/NaSoCRCd.pdf (2007).

32. Bakx R, Visser O, Josso J, Meijer S, Slors JF, van Lanschot JJ. Management of recurrent rectal cancer: a population based study in greater Amsterdam. World J Gastroenterol. 2008;14:6018-23.

33. Sauer R, Becker H, Hohenberger W; German Rectal Cancer Study Group, et al. Preoperative versus postoperative chemoradiotherapy for rectal cancer. $N$ Engl J Med. 2004;351:1731-40.

34. Frykholm GJ, Glimelius B, Påhlman L. Preoperative or postoperative irradiation in adenocarcinoma of the rectum: final treatment results of a randomized trial and an evaluation of late secondary effects. Dis Colon Rectum. 1993;36:564-72. 